\title{
Optimal Linear Precoding for Indoor Visible Light Communication System
}

\author{
Houssem Sifaou, Ki-Hong Park, Abla Kammoun, Mohamed-Slim Alouini \\ Computer, Electrical, and Mathematical Sciences \& Engineering Division, \\ King Abdullah University of Science and Technology, Thuwal, Saudi Arabia
}

\begin{abstract}
Visible light communication (VLC) is an emerging technique that uses light-emitting diodes (LED) to combine communication and illumination. It is considered as a promising scheme for indoor wireless communication that can be deployed at reduced costs while offering high data rate performance. In this paper, we focus on the design of the downlink of a multi-user VLC system. Inherent to multi-user systems is the interference caused by the broadcast nature of the medium. Linear precoding based schemes are among the most popular solutions that have recently been proposed to mitigate inter-user interference. This paper focuses on the design of the optimal linear precoding scheme that solves the max-min signal-tointerference-plus-noise ratio (SINR) problem. The performance of the proposed precoding scheme is studied under different working conditions and compared with the classical zero-forcing precoding. Simulations have been provided to illustrate the high gain of the proposed scheme.

Index Terms-Visible light communication, multi-user multiple-input multiple-output system, optimal linear precoding, max-min SINR.
\end{abstract}

\section{INTRODUCTION}

Recently, we have witnessed an increasing interest in the technology of visible light communications (VLC) [1], [2], as a result of the recent advances in the fabrication of light emitting diodes (LEDs). VLC uses white LEDs that transmit data by changing the light intensity; but variations of the modulated optical signal cannot be noticed by the human eyes which perceive only the average light intensity. Due to its main advantages, such as ease of deployment and low cost, VLC is now being considered as a potential candidate to complement conventional indoor radio frequency (RF) communications.

The use of multiple-input-multiple-output (MIMO) techniques appears to be natural in VLC systems as illumination usually requires the use of multiple LEDs. This excess in the number of degrees of freedom offered by the availability of multiple transceivers can be leveraged to ensure high data rates [3]-[6]. Very recently, the use of multi-user MIMO (MUMIMO) techniques for VLC systems has been studied [5], [6], where the issue of mitigating the inter-user interference has been addressed. Towards this goal, linear precoding schemes aiming at minimizing the mean square error (MSE) [6] or maximizing the signal-to-interference-plus-noise ratio (SINR) [5] have been proposed.

In this paper, we consider the problem of designing the optimal linear precoding (OLP) that solves the max-min SINR problem in MU-MIMO VLC systems. Such a problem has been widely investigated in RF MU-MIMO systems [7]-[10]. However, the results of these works could not be applied

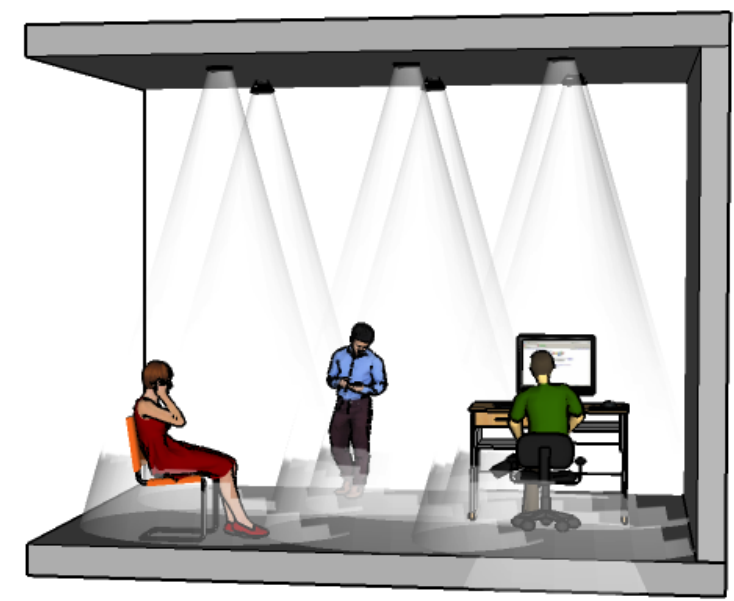

Fig. 1: Indoor VLC system

since they do not take into consideration many practical considerations of VLC systems. As a matter of fact, timedomain signals in VLC are real-valued and positive, while RF counterparts are complex. Moreover, a constraint on the average optical power should be considered in VLC to meet with technical illumination requirements.

This paper derives the optimal linear precoding that solves the max-min SINR problem and compare its performance with the previously proposed Zero forcing (ZF) precoding in [5]. We show that our proposed precoding provides a significant gain in performance especially when the users are close to each other and the inter-user interference is high.

The remainder of the paper is organized as follows. The next section introduces the system model. In section III, the OLP is designed. Before concluding in section $\mathrm{V}$, numerical results are given in section IV.

\section{SySTEM MODEL}

We consider a MU-MIMO VLC system where $M$ transmitters communicate with $K$ user equipments (UEs) $(M>K)$ as shown in Fig. 1. Each UE is equipped with single receiving unit. Intensity modulation is employed at the transmitter and direct detection at the receiver. The transmitting LEDs produce light intensity proportional to the input electric signal and the receiver converts the received light intensity into electric signal. The light intensity variation can not be detected by the human eyes which perceive only the average light intensity. 
Taking into account the required level of illumination, the characteristics of LEDs and the presence of intensity modulation, three constraints on the input electric signal $y_{n}$ have to be considered:

- The input electric signal must be real valued and positive: $y_{n} \geq 0$.

- The input electric signal $y_{n}$ must be lower than a value $p_{\max }$ in order to ensure that the LED works in its linear dynamic range [6]: $y_{n} \leq p_{\max }$.

- The expectation of the input electric signal must be equal to a constant determined by the illumination level: $\mathbb{E}\left(y_{n}\right)=p_{n}, \quad \forall n=1, \cdots, M[11]$.

Let $s_{k}$ be the symbol intended to UE $k$. We assume that $s_{k} \in[-1,1]$ with zero mean. Let $\mathbf{W}=\left[w_{n, k}\right] \in \mathbb{R}^{M \times K}$ denotes the precoding matrix. The transmitted signal at the $n$-th transmitting unit is:

$$
x_{n}=\sum_{k=1}^{K} s_{k} w_{n, k}
$$

In order to satisfy the constraint $\mathbb{E}\left(y_{n}\right)=p_{n}, \forall n$, a DC offset $p_{n}$ should be added to $x_{n}$.

$$
y_{n}=x_{n}+p_{n} .
$$

Since $s_{k} \in[-1,1]$, we have

$$
-\sum_{k=1}^{K}\left|w_{n, k}\right|+p_{n} \leq y_{n} \leq \sum_{k=1}^{K}\left|w_{n, k}\right|+p_{n}, \quad \forall n .
$$

To ensure that $y_{n}$ is in the linear dynamic range of the LEDs, we should satisfy

$$
\sum_{k=1}^{K}\left|w_{n, k}\right|+p_{n} \leq p_{\max }
$$

Under the assumption of positivity of the input signal

$$
-\sum_{k=1}^{K}\left|w_{n, k}\right|+p_{n} \geq 0
$$

The two constraints in (4) and (5) can be combined as:

$$
\sum_{k=1}^{K}\left|w_{n, k}\right| \leq \tilde{p}_{n}
$$

where $\tilde{p}_{n}=\min \left(p_{n}, p_{\max }-p_{n}\right)$.

For VLC, the line of sight propagation dominates [4], [12] and the channel gain from the $n$-th transmitter to the $k$-th receiver can be expressed as [4], [13]:

$$
h_{n, k}=\left\{\begin{aligned}
\frac{\rho A}{i_{n, k}^{2}} R\left(\phi_{n, k}\right) \cos \left(\theta_{n, k}\right), & \theta_{n, k} \leq \theta_{c} \\
0, & \theta_{n, k}>\theta_{c}
\end{aligned}\right.
$$

where $\phi_{n, k}$ is the angle of emission with respect to the $n$-th transmitter, $\theta_{n, k}$ is the incident angle with respect to the $k$-th receiver, $i_{n, k}$ is the distance that separates the $n$-th transmitter and the $k$-th receiver, $\theta_{c}$ is the receiver filed of view (FOV), $\rho$ is the photo detector (PD) responsivity and $A$ is the collection area given by:

$$
A=\frac{q^{2}}{\sin ^{2}\left(\theta_{c}\right)} A_{P D}
$$

where $q$ is the refractive index of optical concentrator and $A_{P D}$ is the PD area. $R\left(\phi_{n, k}\right)$ is the Lambertian radiant intensity:

$$
R\left(\phi_{n, k}\right)=\frac{(m+1) \cos ^{m}\left(\phi_{n, k}\right)}{2 \pi},
$$

where $m$ is the order of the Lambertian emission mode number [4], [13]. Let $\mathbf{h}_{k}=\left[h_{1, k}, \cdots, h_{M, k}\right]^{T}$ be the channel vector corresponding to the $k$-th UE. After removing the DC offset $p_{n}$ introduced at the transmitter, the useful received signal at the $k$-th UE can be expressed as:

$$
r_{k}=\mathbf{h}_{k}^{T} \mathbf{w}_{k} s_{k}+\sum_{j \neq k} \mathbf{h}_{k}^{T} \mathbf{w}_{j} s_{j}+z_{k},
$$

where $z_{k}$ is the additive noise. In VLC, $z_{k}$ is assumed to be real valued Gaussian distributed with zero mean and variance $\sigma_{k}^{2}$ [4], [13]:

$$
\sigma_{k}^{2}=2 e P_{s, k} B+2 e \rho \xi_{a m b} A 2 \pi\left(1-\cos \left(\theta_{c}\right)\right) B+i_{a m p}^{2} B
$$

where $e$ is the electronic charge, $B$ is the bandwidth, $\xi_{a m b}$ is the ambient photocurrent, $i_{a m p}$ is the preamplifier noise density and $P_{s, k}$ is defined as:

$$
P_{s, k}=\sum_{n=1}^{M} p_{n} h_{n, k}
$$

For notational convenience, we define $\boldsymbol{\sigma}=\left[\sigma_{1}, \cdots, \sigma_{K}\right]^{T}$. The vector $\mathbf{r}$ collecting the received signals at the UEs can be expressed as:

$$
\mathbf{r}=\mathbf{H W} \mathbf{s}+\mathbf{z}
$$

where $\mathbf{H} \in \mathbb{R}^{K \times M}$ is the channel matrix, $\mathbf{s}=\left[s_{1}, \cdots, s_{K}\right]^{T}$ denotes the symbol vector and $\mathbf{z}=\left[z_{1}, \cdots, z_{K}\right]^{T}$ is the noise vector.

\section{PRECODING DESIGN}

In this section, we address the problem of designing the optimal linear precoding (OLP) that solves the max-min SINR problem while satisfying the optical power constraint in (6). Due to complexity concerns, the linear precoding scheme are generally preferred to the nonlinear ones. Prior to presenting our proposed scheme, we shall review the classical zeroforcing $(\mathrm{ZF})$ scheme, which will be used later for comparison.

\section{A. Zero-forcing $(Z F)$ :}

The ZF precoding matrix is defined as:

$$
\mathbf{W}=\mathbf{H}^{T}\left(\mathbf{H H}^{T}\right)^{-1} \operatorname{diag}(\boldsymbol{\gamma}),
$$

where $\gamma=\left[\gamma_{1}, \cdots, \gamma_{K}\right]^{T}$ and $\gamma_{k}$ is the symbol gain for UE $k$. The $\mathrm{ZF}$ precoding suppresses the interference and the received signal can be expressed as:

$$
r_{k}=\gamma_{k} s_{k}+z_{k}
$$


The optimal symbol gain vector $\gamma$ has been determined in [5] where it has been shown that:

$$
\gamma_{k}^{\star}=\sigma_{k} \mu_{k}^{\star}
$$

where $\mu_{k}^{\star}=\min _{n} \frac{\tilde{p}_{n}}{\left(\mathbf{A} \mathbf{1}_{K}\right) n}$ and $\mathbf{A}=$ $\operatorname{abs}\left(\mathbf{H}^{T}\left(\mathbf{H H}^{T}\right)^{-1}\right) \operatorname{diag}(\boldsymbol{\sigma}) . \quad \mathbf{1}_{K}$ is the all-one vector of size $K$.

\section{B. Optimal linear precoding:}

In this section, we propose to determine the optimal linear precoding that solves the following max-min SINR problem:

$$
\begin{aligned}
& \mathcal{P}: \max _{\mathbf{W}} \min _{k} \operatorname{SINR}_{k} \\
& \text { subject to } \sum_{k=1}^{K}\left|w_{n, k}\right| \leq \tilde{p}_{n}, \quad n=1, \cdots, M
\end{aligned}
$$

where $\mathrm{SINR}_{k}$ is given by:

$$
\mathrm{SINR}_{k}=\frac{\left|\mathbf{h}_{k}^{T} \mathbf{w}_{k}\right|^{2}}{\sum_{j \neq k}\left|\mathbf{h}_{k}^{T} \mathbf{w}_{j}\right|^{2}+\sigma_{k}^{2}}
$$

Problem $(\mathcal{P})$ can be rewritten as

$$
\begin{array}{rl}
\mathcal{P}_{1}: \max _{\mathbf{W}, t} & t \\
\text { subject to } & \sum_{k=1}^{K}\left|w_{n, k}\right| \leq \tilde{p}_{n}, \quad n=1, \cdots, M \\
& \frac{\left|\mathbf{h}_{k}^{T} \mathbf{w}_{k}\right|^{2}}{\sum_{j \neq k}\left|\mathbf{h}_{k}^{T} \mathbf{w}_{j}\right|^{2}+\sigma_{k}^{2}} \geq t, \quad k=1, \cdots, K .
\end{array}
$$

To solve $\mathcal{P}_{1}$, we shall first rewrite the constraints in a different form. The power constraints in $\left(\mathcal{P}_{1}\right)$ can be rewritten as :

$$
\left\|\mathbf{W}^{T} \mathbf{e}_{n}\right\|_{1} \leq \tilde{p}_{n}, \quad n=1, \cdots, M,
$$

where $\mathbf{e}_{n}$ is the all zero vector having the $n$-th element equal to 1 . The $L_{1}$ norm constraints can be transformed into the following linear constraints:

$$
-\mathbf{a}_{n} \leq \mathbf{W}^{T} \mathbf{e}_{n} \leq \mathbf{a}_{n}, \quad \mathbf{1}_{K}^{T} \mathbf{a}_{n} \leq \tilde{p}_{n}, \quad n=1, \cdots, M,
$$

where $\mathbf{a}_{n} \in \mathbb{R}^{K}$ is a new optimization variable. In order to simplify further the optimization problem, we introduce the following vectors : $\mathbf{w}=\operatorname{vec}\left(\mathbf{W}^{T}\right)=\left[\mathbf{e}_{1}^{T} \mathbf{W}, \cdots, \mathbf{e}_{M}^{T} \mathbf{W}\right]^{T}$ and $\mathbf{a}=\left[\mathbf{a}_{1}^{T}, \cdots, \mathbf{a}_{M}^{T}\right]^{T}$. Using these notations, the power constraints in (9) can be written as:

$$
-\mathbf{a} \leq \mathbf{w} \leq \mathbf{a}, \quad \mathbf{U} \mathbf{a} \leq \tilde{\mathbf{p}},
$$

where $\mathbf{U}=\mathbf{I}_{M} \otimes \mathbf{1}_{K}^{T}$ and $\tilde{\mathbf{p}}=\left[\tilde{p}_{1}, \cdots, \tilde{p}_{M}\right]^{T}, \mathbf{I}_{M}$ being the identity matrix of size $M$ and $\otimes$ denoting the Kronecker product. We now work out the SINR constraints of $\left(\mathcal{P}_{1}\right)$ :

$$
\sum_{j \neq k}\left|\mathbf{h}_{k}^{T} \mathbf{w}_{j}\right|^{2}+\sigma_{k}^{2} \leq \frac{1}{t}\left|\mathbf{h}_{k}^{T} \mathbf{w}_{k}\right|^{2}, \quad \forall k,
$$

to express them in terms of vector $\mathbf{w}$. The left hand side can be rewritten using $L_{2}$ norm as: ${ }^{1}$

$$
\left\|\begin{array}{l}
\mathbf{W}_{k}^{T} \mathbf{h}_{k} \\
\sigma_{k}
\end{array}\right\|_{2} \leq \frac{1}{\sqrt{t}} \mathbf{h}_{k}^{T} \mathbf{w}_{k}, \quad \forall k,
$$

where $\mathbf{W}_{k}$ is the matrix obtained from $\mathbf{W}$ by removing the $k$-th column. Let $\mathbf{I}_{K}^{k}$ denotes the matrix obtained from identity matrix of size $K$ by setting the $(k, k)$-th element to zero. Then:

$$
\begin{aligned}
\mathbf{W}_{k}^{T} \mathbf{h}_{k} & =\mathbf{I}_{K}^{k} \mathbf{W}^{T} \mathbf{h}_{k} \\
& =\operatorname{vec}\left(\mathbf{I}_{K}^{k} \mathbf{W}^{T} \mathbf{h}_{k}\right) \\
& =\left(\mathbf{h}_{k}^{T} \otimes \mathbf{I}_{K}^{k}\right) \mathbf{w}
\end{aligned}
$$

and

$$
\begin{aligned}
\mathbf{w}_{k} & =\operatorname{vec}\left(\mathbf{w}_{k}^{T}\right)=\operatorname{vec}\left(\mathbf{e}_{k}^{T} \mathbf{W}\right) \\
& =\left(\mathbf{I}_{M} \otimes \mathbf{e}_{k}^{T}\right) \operatorname{vec}\left(\mathbf{W}^{T}\right)=\left(\mathbf{I}_{M} \otimes \mathbf{e}_{k}^{T}\right) \mathbf{w}
\end{aligned}
$$

Thus, the constraints in (10) can be reformulated as:

$$
\left\|\mathbf{B}_{k} \mathbf{w}+\boldsymbol{\sigma}_{k}\right\|_{2} \leq \frac{1}{\sqrt{t}} \mathbf{h}_{k}^{T}\left(\mathbf{I}_{M} \otimes \mathbf{e}_{k}^{T}\right) \mathbf{w}, \quad \forall k,
$$

with $\mathbf{B}_{k} \in \mathbb{R}^{(K+1) \times M K}$ and $\boldsymbol{\sigma}_{k} \in \mathbb{R}^{(K+1)}$ are given by:

$$
\mathbf{B}_{k}=\left[\begin{array}{l}
\mathbf{h}_{k}^{T} \otimes \mathbf{I}_{K}^{k} \\
\mathbf{0}_{1 \times M K}
\end{array}\right], \quad \boldsymbol{\sigma}_{k}=\left[0, \cdots, 0, \sigma_{k}\right]^{T} .
$$

For fixed $t$, the reformulated SINR constraints in (11) are second-order cone constraints which are convex [14]-[16]. Our optimization problem $\mathcal{P}_{1}$ turns out to be quasi-convex and can be solved using the bisection algorithm [17]. Each iteration of the bisection algorithm consists in holding $t$ fixed and solving for $\mathbf{w}$ the following feasibility problem,

find $\mathbf{w}$

subject to $\left\|\mathbf{B}_{k} \mathbf{w}+\boldsymbol{\sigma}_{k}\right\|_{2} \leq \frac{1}{\sqrt{t}} \mathbf{h}_{k}^{T}\left(\mathbf{I}_{M} \otimes \mathbf{e}_{k}^{T}\right) \mathbf{w}, \quad \forall k$

$$
-\mathbf{a} \leq \mathbf{w} \leq \mathbf{a}, \quad \mathbf{U a} \leq \tilde{\mathbf{p}}
$$

which is a second-order cone program (SOCP) that can be efficiently solved using CVX [18]. The optimal $t$ corresponds thus to the maximum value for which it exists $\mathbf{w}$ satisfying the constraints in (12). To sum up, solving $\mathcal{P}_{1}$ can be performed using the following algorithm:

\footnotetext{
${ }^{1}$ Without loss of optimality, we can assume that $\mathbf{h}_{k}^{T} \mathbf{w}_{k} \geq 0$ since the objective function and the constraints are invariant to sign changes of $\mathbf{w}_{k}$.
} 


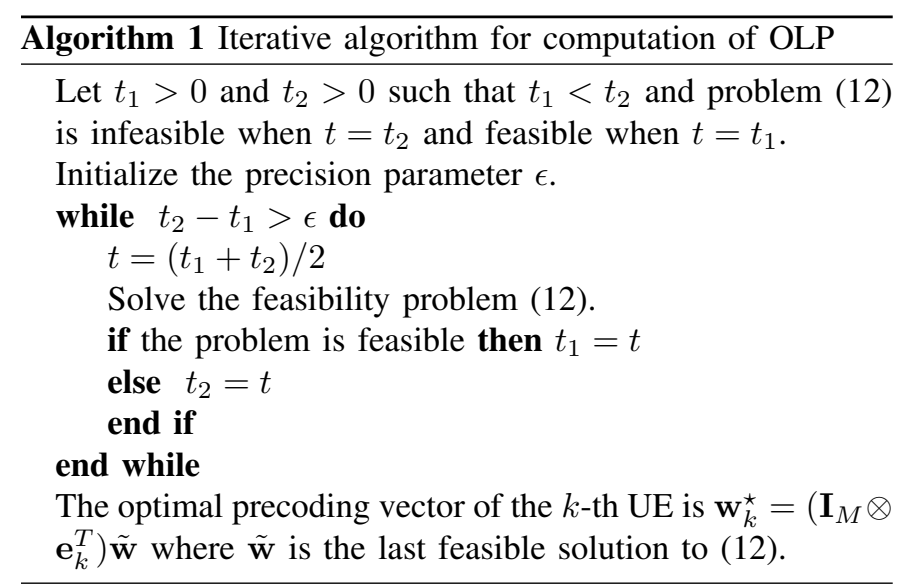

Algorithm 1 converges in exactly $\log _{2}\left[\left(t_{2}-t_{1}\right) / \epsilon\right]$ iterations. In order to accelerate the convergence of Algorithm 1, the initial values $t_{1}$ and $t_{2}$ can be determined efficiently using the following algorithm:

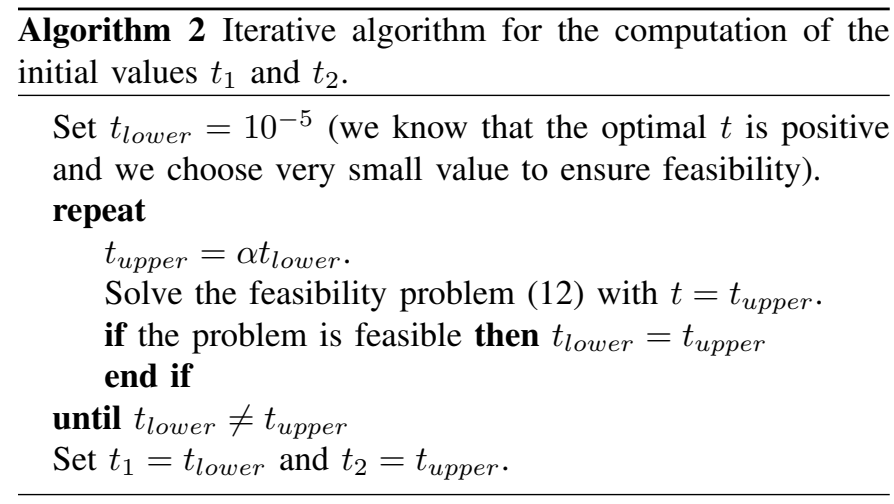

where $\alpha$ is a scale factor strictly greater than 1 .

Remark 1. The overall complexity of the precoding design is governed by the complexity of Algorithm 1 and Algorithm 2. For Algorithm 1, the number of iterations is given by $\log _{2}\left[\left(t_{2}-t_{1}\right) / \epsilon\right]$ and as such is low when the difference $t_{2}-t_{1}$ is small. On the other hand, Algorithm 2 would require less iterations as $\alpha$ increases. However, increasing $\alpha$ produces higher values for the difference $t_{2}-t_{1}$, and as a consequence increases the complexity of Algorithm 1. That being said, it is worth mentioning that Algorithm 1 and Algorithm 2 are used at the pace of the change of the channel, which is slow as far as VLC applications are concerned.

\section{Numerical Results}

In this section, the proposed OLP is compared with the classical ZF precoding using simulations. We consider a VLC system composed of 6 transmitters installed on the ceiling of the room as depicted in Fig. 1. The positions of the transmitters are given in Fig. 2. We assume that UEs are static, which allow us to consider static channels. The average powers for all transmitters $\left\{p_{n}\right\}$ are assumed to be the same and equal to $p$ where, for simulation purposes, $p$ is assumed to range between $15 \mathrm{dBm}$ and $30 \mathrm{dBm}$. Without loss of generality, we assume

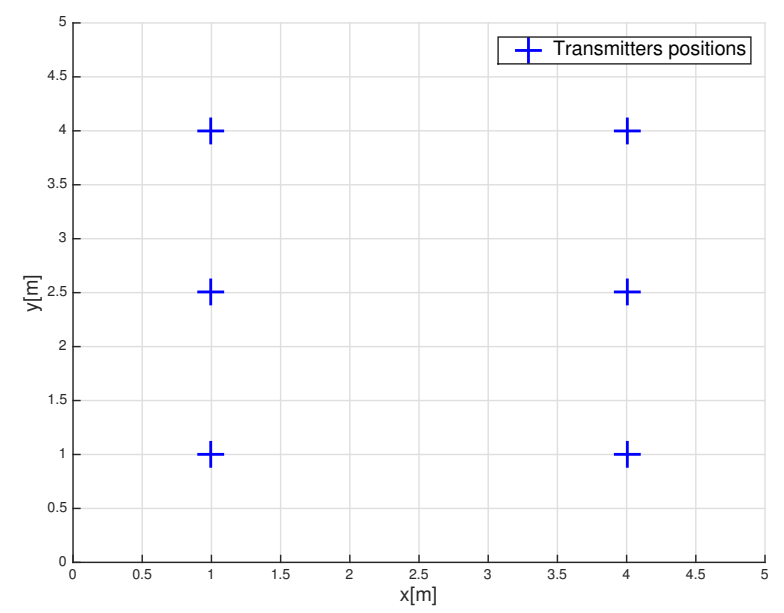

Fig. 2: Positions of the transmitters on the roof.

also that $p_{\max }-p_{n} \gg p_{n}$ and as such $\tilde{p}_{n}=\min \left(p_{n}, p_{\max }-\right.$ $\left.p_{n}\right)=p_{n}$. The VLC system parameters are summarized in Table 1. The performance measure is the rate per UE $r$ defined as:

$$
r=\frac{1}{K} \sum_{k=1}^{K} B \log _{2}\left(1+\operatorname{SINR}_{k}\right) .
$$

TABLE 1: VLC system parameters.

\begin{tabular}{|l|l|}
\hline Room Size & $5 m \times 5 m \times 3 m$ \\
\hline Mode number $m$ of Lambertian emission & 1 \\
\hline Photo Detector reponsivity $\rho$ & $0.4 \mathrm{~A} / \mathrm{W}$ \\
\hline Photo Detector area $A_{P D}$ & $1 \mathrm{~cm}^{2}$ \\
\hline Receiver FOV $\theta_{c}$ & $60 \mathrm{deg}$ \\
\hline Refractive index of optical concentrator $q$ & 1.5 \\
\hline Pre-amplifier noise density $i_{a m p}$ & $5 \mathrm{pA} / \mathrm{Hz}^{-1 / 2}$ \\
\hline Ambient light photocurrent $\xi_{a m b}$ & $10.93 \mathrm{~A} / \mathrm{m}^{2} / \mathrm{Sr}$ \\
\hline Bandwidth $B$ & $100 \mathrm{MHz}$ \\
\hline
\end{tabular}

In Fig. 3, we compare the rate per UE vs. the power constraint of the proposed OLP with that of the classical ZF precoding for two different scenarios depending on the separation between the UEs. Namely, the first scenario corresponds to the case where the UEs are positioned far away from each other, while the second scenario studies the situation in which UEs become closer. The positions of UEs in both scenarios are indicated in Table 2 and Table 3 respectively. As seen from Fig. 3, the performance of the OLP is better and the improvement in performance becomes higher when the UEs are close to each other or the power constraint $p$ is low (low SNR regime).

The impact of the number of UEs is investigated in Fig. 4 , where the rate per UE vs. $p$ is plotted for up to 5 UEs positioned randomly in the room. As seen, regardless of the number of UEs, our proposed precoding achieves a significant gain as compared to the ZF precoding especially in the low SNR regime. 


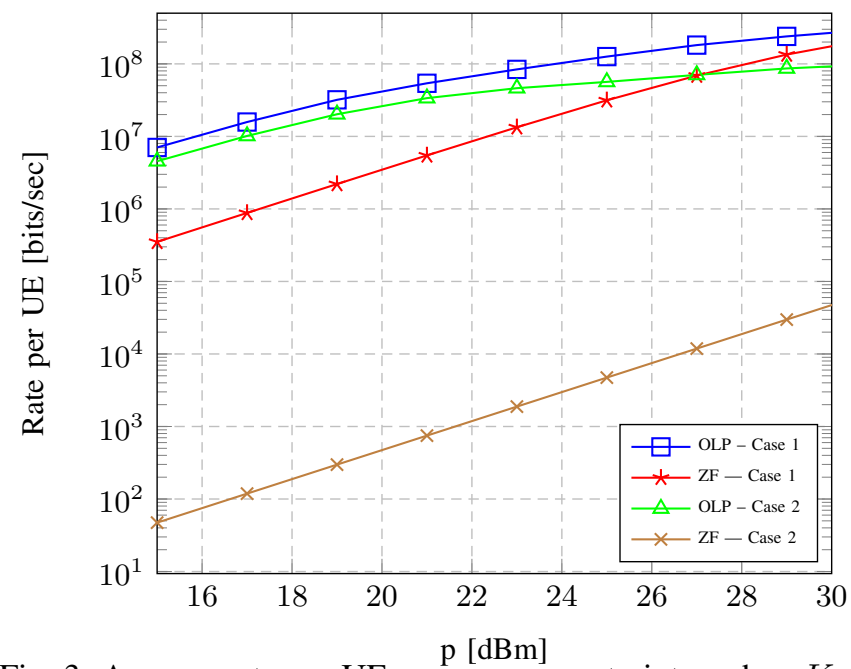

Fig. 3: Average rate per UE vs. power constraint $p$ when $K=$ 4.

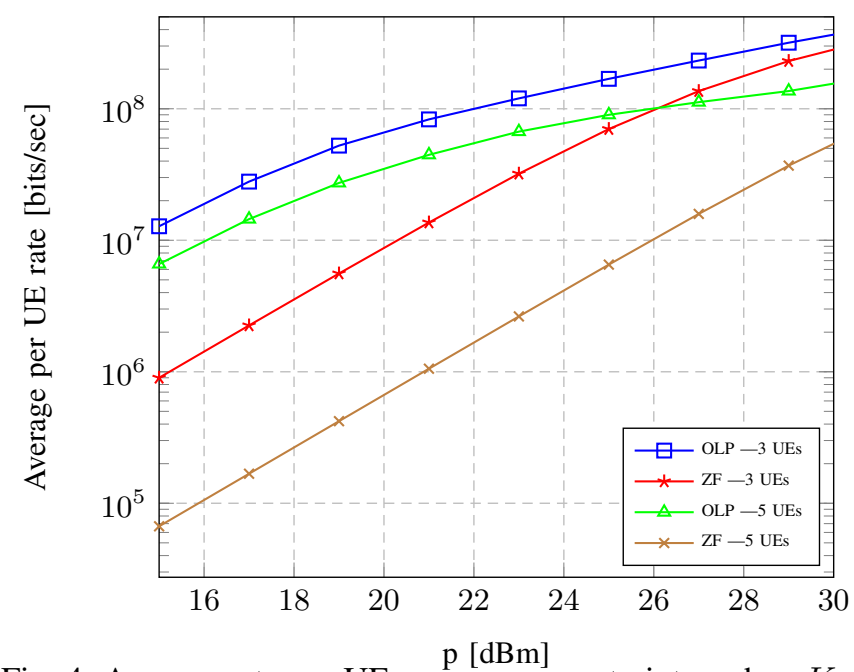

Fig. 4: Average rate per UE vs. power constraint $p$ when $K=$ 3 and $K=5$.

TABLE 2: UEs with large separation - Case 1.

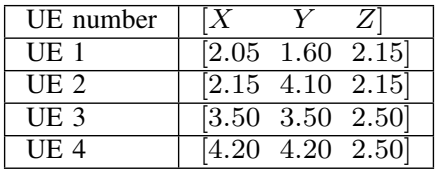

TABLE 3: UEs with small separation - Case 2.

\begin{tabular}{|c|c|c|}
\hline UE number & {$[X$} & $Z]$ \\
\hline UE 1 & {$\left[\begin{array}{ll}2.05 & 2.20\end{array}\right.$} & $2.15]$ \\
\hline UE 2 & {$\left[\begin{array}{ll}2.05 & 2.40\end{array}\right.$} & $2.15]$ \\
\hline UE 3 & {$\left[\begin{array}{ll}2.05 & 2.60\end{array}\right.$} & $2.15]$ \\
\hline UE 4 & {$\left[\begin{array}{ll}2.05 & 2.80\end{array}\right.$} & $2.15]$ \\
\hline
\end{tabular}

\section{CONCLUSION}

In this paper, we considered the problem of precoding design for indoor VLC systems. We have determined the optimal linear precoding that solves the max-min SINR problem. It has been shown by simulation that our proposed technique provides a significant gain in performance compared to the classical ZF precoding especially in the scenario where the UEs are close to each other.

\section{REFERENCES}

[1] D. Tsonev, H. Chun, S. Rajbhandari, J. J. D. McKendry, S. Videv, E. Gu, M. Haji, S. Watson, A. E. Kelly, G. Faulkner, M. D. Dawson, H. Haas, and D. O'Brien, “A 3-Gb/s single-LED OFDM-based wireless VLC link using a Gallium Nitride $\mu$ LED," IEEE Photonics Technology Letters, vol. 26, no. 7, pp. 637-640, Apr 2014.

[2] D. Tsonev, S. Videv, and H. Haas, "Towards a $100 \mathrm{~Gb} / \mathrm{s}$ visible light wireless access network," Opt. Express, vol. 23, no. 2, pp. 1627-1637, Jan 2015 .

[3] D. Takase and T. Ohtsuki, "Optical wireless MIMO communications (OMIMO)," in Proc. IEEE GLOBECOM, Texas, USA, Nov 2004, vol. 2 , pp. 928-932.

[4] L. Zeng, D. C. O’Brien, H. L. Minh, G. E. Faulkner, K. Lee, D. Jung, Y. Oh, and E. T. Won, "High data rate multiple input multiple output (MIMO) optical wireless communications using white led lighting," IEEE Journal on Selected Areas in Communications, vol. 27, no. 9, pp. 1654-1662, Dec 2009.

[5] Z. Yu, R. J. Baxley, and G. T. Zhou, "Multi-user MISO broadcasting for indoor visible light communication," in IEEE International Conference on Acoustics, Speech and Signal Processing, Vancouver, Canada, May 2013, pp. 4849-4853.

[6] B. Li, J. Wang, R. Zhang, H. Shen, C. Zhao, and L. Hanzo, "Multiuser MISO transceiver design for indoor downlink visible light communication under per-LED optical power constraints," IEEE Photonics Journal, vol. 7, no. 4, pp. 1-15, Aug 2015.

[7] D. W. H. Cai, T. Q. S. Quekand, C. Wei Tan, and S. H. Low, "Maxmin SINR coordinated multipoint downlink transmission - duality and algorithms," IEEE Transactions on Signal Processing, vol. 60, pp. 53845395, 2012.

[8] H. Sifaou, A. Kammoun, L. Sanguinetti, M. Debbah, and M.-S. Alouini, "Max-min SINR low complexity transceiver design for single cell massive MIMO," in 2016 IEEE 17th International Workshop on Signal Processing Advances in Wireless Communications (SPAWC), Edinburgh, UK, Jul 2016, pp. 1-6.

[9] H. Sifaou, A. Kammoun, L. Sanguinetti, M. Debbah, and M.-S. Alouini, "Max-min SINR in large-scale single-cell MU-MIMO: Asymptotic analysis and low complexity transceivers," IEEE Trans. Signal Processing, vol. 65, no. 7, pp. 1841-1854, Apr 2017.

[10] K. Cumanan, L. Musavian, S. Lambotharan, and A. B. Gershman, "SINR balancing technique for downlink beamforming in cognitive radio networks," IEEE Signal Processing Letters, vol. 17, no. 2, pp. 133-136, Feb 2010.

[11] J. B. Wang, Q. S. Hu, J. Wang, M. Chen, and J. Y. Wang, "Tight bounds on channel capacity for dimmable visible light communications," Journal of Lightwave Technology, vol. 31, no. 23, pp. 3771-3779, Oct 2013.

[12] T. Komine and M. Nakagawa, "Fundamental analysis for visiblelight communication system using LED lights," IEEE Transactions on Consumer Electronics, vol. 50, no. 1, pp. 100-107, Feb 2004.

[13] J. M. Khan and J. R. Barry, "Wireless infrared communications," Proceedings of the IEEE, vol. 85, no. 2, pp. 265-298, 1997.

[14] A. Wiesel, Y. Eldar, and S. Shamai, "Linear precoding via conic optimization for fixed MIMO receivers," IEEE Transactions on Signal Processing, vol. 54, pp. 161-176, 2006.

[15] M. Bengtsson and B. Ottersten, "Optimal and suboptimal transmit beamforming," in Handbook of Antennas in Wireless Communications. L. C. Godara, Ed CRC Press, 2001.

[16] W. Yu and T. Lan, "Transmitter optimization for the multi-antenna downlink with per-antenna power constraints," IEEE Trans. Signal Processing, vol. 55, no. 6-1, pp. 2646-2660, 2007.

[17] S. Boyd and L. Vandenberghe, Convex Optimization, Cambridge University Press, New York, 2004.

[18] Inc. CVX Research, "CVX: Matlab software for disciplined convex programming, version 2.0 beta," Sept. 2012. 